Accepted for publication in Language, Cognition and Neuroscience @ 2021 Taylor \& Francis, https://www.tandfonline.com/toc/plcp21/current. This article may not exactly replicate the final published version. It is not the copy of record.

\title{
Age-related effects on lexical, but not syntactic, processes during sentence production
}

Sophie M. Hardy ${ }^{\mathrm{a}, \mathrm{b}^{*}}$, Katrien Segaert ${ }^{\mathrm{a}}$, and Linda Wheeldon ${ }^{\mathrm{c}}$

${ }^{\text {a }}$ Centre for Human Brain Health, School of Psychology, University of Birmingham, UK

${ }^{\mathrm{b}}$ Department of Psychology, University of Warwick, UK

${ }^{c}$ Department of Foreign Languages and Translation, University of Agder, Norway

* Corresponding author: Department of Psychology, University of Warwick, Coventry, CV4

7AL, UK. Email: sophie.hardy@warwick.ac.uk. ORCiD ID: https://orcid.org/0000-0002-

0961-8873. 


\begin{abstract}
We investigated the effect of healthy ageing on the lexical and syntactic processes involved in sentence production. Young and older adults completed a semantic interference sentence production task: we manipulated whether the target picture and distractor word were semantically related or unrelated and whether they fell within the same phrase ( "the watch and the clock/hippo move apart") or different phrases ("the watch moves above the clock/hippo"). Both age groups were slower to initiate sentences containing a larger, compared to a smaller, initial phrase, indicating a similar phrasal scope of advanced planning. However, older adults displayed significantly larger semantic interference effects (slower to initiate sentences when the target picture and distractor word were related) than young adults, indicating an age-related increase in lexical competition. Thus, while syntactic planning is preserved with age, older speakers encounter problems managing the temporal co-activation of competing lexical items during sentence production.
\end{abstract}

Keywords: healthy ageing, sentence production, syntactic planning, lexical competition, lexical retrieval, semantic interference, picture-word interference. 


\section{Introduction}

Successful communication requires the conceptualisation of a pre-verbal message and the formulation of a corresponding utterance (Bock \& Levelt, 1994; Levelt, 1989). At the sentence level, the formulation process involves the rapid retrieval of lexical items and the generation of an appropriate syntactic structure, which must be integrated correctly to convey the intended message. As we age, cognitive and neuroanatomical changes occur that create challenges for language processing, which may in turn lead to age-related changes in the processes involved in speech planning and production (see Burke \& Shafto, 2008, for a review). In this study, we investigated how the lexical and syntactic processes involved in sentence production are affected by healthy ageing.

Despite the cognitive and neuroanatomical changes associated with ageing, there is not a straightforward relationship between healthy ageing and language decline; instead the relationship is complex with some language skills being more negatively affected by ageing than others, and some skills being preserved (Burke \& Shafto, 2008; Peelle, 2019; Wingfield \& Grossman, 2006). This contrast between decline and preservation is evident at both the word and sentence level of language production in experimental settings and in more naturalistic contexts. For example, while older adults may experience increased tip-of-thetongue states (when a speaker is certain that they know a word, but is unable to produce it; Burke et al., 1991; Segaert et al., 2018), vocabulary size and knowledge typically increase with age (Verhaeghen, 2003). Likewise, at the sentence level, ageing is associated with an increase in syntactic errors, such as the use of the incorrect tense (Kemper et al., 2001, 2003, 2004; Rabaglia \& Salthouse, 2011), but older adults do maintain the ability to switch between different syntactic alternatives and to align their syntactic choices with others in dialogue (Davidson et al., 2003; Hardy et al., 2017). Investigating how different aspects of language are affected by healthy ageing is therefore critical for better understanding the multi-factorial 
nature of language processing in old age. The aim of our study was to investigate how older adults' lexical and syntactic processing is affected by the co-activation of semantic competitors during sentence production.

When a word is selected for production, lexical representations of semantically similar words (e.g., cat-dog) are also activated (Dell, 1986; Roelofs, 1992). The exact nature of this spreading activation architecture is debated (see Roelofs \& Ferreira, 2019, for a review), but, in order to maintain speech fluency, a speaker must prevent the activation of a semantic competitor from interfering with lexical retrieval and speech production. The ability to ignore distracting and irrelevant information typically declines with age (Tipper, 1991; Tun et al., 2002; Weeks \& Hasher, 2014). It follows therefore that older adults may experience increased interference from semantic distractors during speech production. However, the evidence is mixed: while some studies have found older adults' speech is slowed due to competition from a near semantic neighbour (Britt et al., 2016; LaGrone \& Spieler, 2006) or auditory distractor (Taylor \& Burke, 2002), others have found no age differences in semantic interference effects during picture naming (Belke \& Meyer, 2007; Burke, 2002; Gordon \& Cheimariou, 2013; Mulatti et al., 2014; Tree \& Hirsh, 2003). Notably, these studies have largely investigated lexical competition effects at the single word level - the findings, therefore, cannot easily be generalised to multiword utterances that are more typical of everyday language production. Indeed, words are rarely produced in isolation; instead they are usually constituent parts of a larger sentence structure (Levelt, 1989). Moreover, Sass et al. (2010) found that the impact of a semantic distractor varies dependent on the speech production context, such that semantic interference effects are considerably stronger during sentence production, compared to single word production. We therefore investigated the effect of semantic interference on older adults' speech production 
in a context where sentences, rather than single words, are produced in order to provide novel insight into the debate surrounding lexical competition and healthy ageing.

One way to investigate semantic interference is the classic picture-word interference paradigm in which a speaker has to name a target picture while ignoring a visually or auditorily presented distractor word (Glaser \& Düngelhoff, 1984; Schriefers et al., 1990; for a recent meta-analytical review, see Bürki et al., 2020). Word reading is a highly automated process in skilled readers in the sense that it cannot be prevented or controlled (LaBerge \& Samuels, 1974; Samuels \& Flor, 1997). ${ }^{1}$ This means that when participants are presented with a written distractor word, they will definitely process it (i.e., access lexical information relating to the word) prior to beginning their naming of the target picture. Consequently, when the presented distractor word and target picture are semantically related, speech onset latencies are slowed because there is increased competition between the two lexical items, which has been attributed to either the lemma level of processing (Dell, 1986; Roelofs, 1992, 1997), the phonological level (Starreveld \& La Heij, 1996) or the post-lexical level (Mahon et al., 2007). Importantly, the picture-word interference paradigm can also be adapted to elicit sentences, instead of single words, thereby making it ideally suited to the investigation of lexical competition during sentence production (Meyer, 1996; Momma et al., 2016; Smith \& Wheeldon, 2004; Yang \& Yang, 2008). Smith and Wheeldon (2004) presented participants with a picture and a written word together on screen that were either semantically related (watch-clock) or unrelated (watch-hippo) and instructed participants to produce sentence descriptions (e.g., "the watch [picture] and the clock [word] move up"). The picture served as the target and was always the first item to be named in the sentence. The written word served

\footnotetext{
${ }^{1}$ The Stroop task in which participants must name the colour of the font, not the written word (e.g., "RED" in blue font), provides strong evidence for the automaticity of reading as participants are significantly slower to name the font colour when it is incongruent with the written text, suggesting that they cannot help themselves from reading (see Augustinova \& Ferrand, 2014, for a review).
} 
as the distractor because, as in the classic picture-word interference task, participants would rapidly read and process the word as soon as it appeared on screen (due to the high automaticity of reading; Augustinova \& Ferrand, 2014; LaBerge \& Samuels, 1974), meaning that they would have accessed lexical information relating to the written word prior to beginning lexical retrieval of the target picture. The task therefore tests how speakers deal with the co-activation of semantic competitors during sentence production.

Smith and Wheeldon (2004) found that speakers were slower to initiate sentences when the target picture and distractor word were semantically related, compared to when they were unrelated. Speech onset latencies are informative about the amount of pre-processing required prior to sentence articulation (Levelt, 1989; Wheeldon, 2013). These findings therefore indicate that there is a temporal overlap of lexical information between different nouns in a to-be-produced sentence - often termed 'horizontal flow' - and that during prearticulatory sentence planning, increased time is required to resolve competition between tobe-produced lexical items that are semantically related. Indeed, horizontal flow of linguistic information is considered to be a vital component of an effective language production system (Rapp \& Samuel, 2002; Wheeldon et al., 2003) and is an important feature of existing theoretical models of speech production (Bock \& Levelt, 1994; Levelt et al., 1999). Similar effects of the horizontal flow of semantic interference have been observed in Mandarin (Yang \& Yang, 2008). Speech onset latencies are also influenced by factors at the syntactic level: speakers take longer to initiate sentences that contain larger, compared to smaller, initial phrases (Hardy et al., 2020; Levelt \& Maassen, 1981; Martin et al., 2010, 2014; Smith \& Wheeldon, 1999). This indicates that speakers engage in a phrasal scope of advanced planning (i.e., plan incrementally in phrasal units) and that a greater amount of pre-planning is required when the initial phrase is larger. Such incremental planning effects are observed for a variety of syntactic constructions of varying linguistic complexity and properties 
97 (Ferreira, 1991; Wagner et al., 2010), and are also evident in Japanese, a head-final language

(Allum \& Wheeldon, 2007). Together, these studies provide evidence that the speed of prearticulatory sentence planning is influenced by the relationship between different lexical items in a sentence, as well as by the size of the initial phrasal unit.

The question remains, however, how sentence planning processes are affected by healthy ageing, and whether lexical competition during sentence planning increases with age. To date, only a handful of studies have investigated on-line sentence planning in older adults using latency measures (Hardy et al., 2020; Spieler \& Griffin, 2006). In a picture description task, Hardy et al. (2020) found that both young and older adults were slower to initiate sentences with larger, compared to smaller, initial phrases, indicating an age-related preservation of syntactic planning scope. However, age group differences did emerge at the lexical level: compared to young adults, older adults displayed less speed benefits due to the picture preview of an upcoming lexical item, and, unlike the young adults, were significantly disadvantaged by the preview (i.e., produced more errors) when its name occurred beyond the initial phrase in the sentence description. This indicates that there are age-related differences in the processes involved in managing the temporal flow of lexical information during sentence planning and, in particular, that older adults are less able to integrate lexical information across phrasal boundaries. Hardy's et al. (2020) study therefore provides the first evidence that healthy ageing affects the lexical, but not syntactic, processes involved in online sentence planning. If lexical processes are indeed more adversely affected than syntactic processes in healthy ageing, older adults should also show increased vulnerability to lexical competition between the words in a sentence. We therefore investigated age-related differences in lexical processing by manipulating the semantic relationship between words (i.e., the picture-word interference sentence production task), which provides a sensitive measure of the temporal flow of lexical information during sentence planning. 


\section{The Present Study}

The aim of this study was to investigate the effects of healthy ageing on sentence planning using an on-line task that taps into the horizontal flow of linguistic information between lexical items. In particular, we employed a semantic interference manipulation to further investigate the divergent effects of old age on lexical and syntactic processing (Hardy et al., 2020). Our study also aimed to provide a novel perspective into age-related effects on lexical competition, which to date has primarily focused on single word production (e.g., Gordon \& Cheimariou, 2013; Taylor \& Burke, 2002). Therefore, in the present study, young and older adults completed a semantic interference sentence production task (similar to Smith \& Wheeldon, 2004) in which we manipulated whether the target picture and distractor word were semantically related or unrelated and whether they were in the same phrase (e.g., "the watch [picture] and the clock/hippo [word] move apart”) or different phrases (e.g., "the watch [picture] moves above the clock/hippo [word]") of the sentence. We recorded speech onset latencies as a measure of the amount of pre-planning that occurred prior to articulation. In line with other studies of semantic interference and/or planning scope using latency measures, we employed a sentence elicitation task involving stimuli movement as this ensured that participants generated specific sentence types, but did so independently and engaged with both syntactic and lexical level processing. Moreover, by removing syntactic choice from our task, we were able to test how exactly participants deal with the early access to lexical information relating to the distractor word (which participants would automatically read and process) that is either contained within the first phrase of the to-be-produced condition). 
\& Wheeldon, 2004), we expect to observe semantic interference effects in participants' speech production (i.e., slowed onset latencies when the target picture and distractor word are related). Critically though this study will address, for the first time, whether age-related differences in lexical competition exist during sentence production. We hypothesise that if lexical competition effects do indeed increase with age (Britt et al., 2016; Taylor \& Burke, 2002), then semantic interference effects will be greater in older adults, compared to young adults. The present study also addresses age-related effects in syntactic planning. If on-line syntactic planning scope is preserved with age (Hardy et al., 2020; Spieler \& Griffin, 2006), we predict that both age groups will plan incrementally in phrasal units, and therefore initiate sentences slower with larger initial phrases (same phrase condition) compared to smaller initial phrases (different phrase condition), as has also been observed in previous studies employing a similar paradigm with young adults (e.g., Martin et al., 2010, 2014; Smith \& Wheeldon, 1999). An alternative hypothesis, however, is that an age-related decline in working memory capacity (particularly at the verbal level; Bopp \& Verhaeghen, 2005) may mean that older adults adopt a more extreme word-by-word sentence planning strategy (i.e., only plan a lexical, not phrasal, unit prior to beginning articulation). Indeed, incremental planning can be strategically controlled by the speaker (e.g., if time pressure is applied; Ferreira \& Swets, 2002) and older adults are known to employ various strategies in other areas of language processing (Altmann \& Kemper, 2006; Stine-Morrow et al., 2008).

Moreover, our experimental design enables us to investigate the influence of phrasal structure on young and older speakers' semantic interference. Critically, if the ability to ignore distracting information and manage the temporal flow of lexical information declines with age (Hardy et al., 2020; Weeks \& Hasher, 2014), we may expect to see age group 
competing lexical items appear within the same or different phrases. This is because, during sentence production, there is a temporal flow of lexical information between to-be-produced lexical items within and across phrasal boundaries (Smith \& Wheeldon, 2004; Yang \& Yang, 2008). Two alternative hypotheses are possible regarding age effects. Firstly, we may observe increased semantic interference for older adults in the same phrase condition since the two competitors are within the same planning unit and are, therefore, processed more closely in time (Wheeldon, 2013). Alternatively, the presentation of the distractor word (which participants will automatically read and process) may lead to the premature activation of lexical information that is not contained within the initial phrase (older adults' preferred scope of planning; Hardy et al., 2020), resulting in a greater semantic interference effect on older adults' speech onset latencies in the different phrase condition.

\section{Method}

\section{Participants}

We recruited 44 young adults ( 32 females; $M=19.7 \mathrm{yrs}, S D=0.8 \mathrm{yrs}$ ) and 46 older adults (28 females; $M=73.1 \mathrm{yrs}, S D=4.9 \mathrm{yrs}$ ). All participants were native English speakers with normal or corrected-to-normal vision, and did not report any language disorders. There was no significant difference in education between age groups. ${ }^{2}$ All older adults scored 26 or above out of $30(M=28.0, S D=1.3)$ on the Montreal Cognitive Assessment (Nasreddine et al., 2005), indicating that they were currently experiencing healthy ageing (scores $<26$ indicate risk of mild cognitive impairment or dementia; Smith et al., 2007). The study was

\footnotetext{
${ }^{2}$ Education was scored according to the International Standard Classification of Education (United Nations, 2011), which classifies education on a scale of 0 (pre-primary school) to 8 (university doctorate). There was no significant difference in scores between young $(M=6.0, S D=0.2)$ and older $(M=5.7, S D=1.4)$ adults, $t(88)=-1.64, p=0.104$. A score of 6.0 indicates engagement in formal education to an undergraduate bachelor level (approximately equal to 17 years).
} 
approved by the University of Birmingham Ethical Review Committee and informed written consent was obtained.

\section{Design}

We used a 2 X 2 X 2 mixed design with one between-participant variable of age group (young vs. older) and two within-participant variables of the semantic relatedness between the target picture and distractor word (related vs. unrelated), and the phrasal structure of the sentence (whether the target picture and distractor word appeared within the same phrase vs. different phrases). In the same phrase condition, a coordinate initial noun phrase was used that contained both lexical items, whereas in the different phrase condition, a simple initial noun phrase was used that only contained the lexical item related to the target picture, and the distractor word appeared within the second phrase (as shown in Figure 1A).

\section{[Figure 1 about here]}

\section{Materials}

The experimental items consisted of 36 photographic pictures and 36 written words of familiar concrete objects. Each picture was paired with a word that was highly semantically related or a near synonym of the corresponding picture name, and with a different word that had no semantic relationship with the picture name: this created 72 picture-word pairs (36 related and 36 unrelated). ${ }^{3}$ We ensured that there was no phonological similarity between the picture name and word within each pair. Each written word served as both a related word in one picture-word pair and as an unrelated word in another pair. This meant that, across all

\footnotetext{
${ }^{3}$ Of the 72 picture-word pairs, 48 matched those used by Smith and Wheeldon (2004). A full stimuli list of the picture-word pairs is available to download online: https://osf.io/rwav9/.
} 
items, the lexical properties of the distractor words, such as frequency and length, were entirely matched between the related and unrelated conditions. Sixteen additional adults (all native English speakers) who did not take part in this study were asked to rate the relatedness of the 72 picture-word pairs on a scale of 0 (not related at all) to 6 (highly related). The related pairs $(M=4.54, S D=0.66)$ were rated as significantly more related than the unrelated pairs $(M=0.31, S D=0.41), t(70)=32.59, p<.001$.

All 72 picture-word pairs each appeared once within the two phrasal structure conditions, creating 144 experimental items. The movement of each picture-word pair was manipulated using E-prime (Schneider et al., 2002), and participants described the movements from left to right using specific sentence types (the target picture always appeared in the leftmost position). In the same phrase condition, the target picture and distractor word moved simultaneously, eliciting a sentence with a coordinate initial noun phrase (The [picture] and the [word] move apart/together"). In the different phrase condition, only the picture moved and the word remained stationary, eliciting a sentence with a simple initial noun phrase ("The [picture] moves above/below the [word]").

We also created 120 fillers from a further 15 pictures and 15 written words in order to increase the variability of the syntactic structures elicited and to reduce predictability about the sentence types. Each filler featured a single picture/word that moved either up, down, left or right (e.g., "The horse moves up"). The fillers contrasted the same phrase items in terms of the complexity of the initial phrase, and contrasted the different phrase items in the total number of noun phrases.

We constructed four blocks that each contained 30 fillers and 36 experimental items (9 per condition). The order of the items was pseudorandomized with the constraint that two consecutive experimental items always featured different pictures and written words and were never of the same phrasal structure. The order of the blocks was rotated across 
participants. Each participant completed a total of 144 experimental items, consisting of 36 items per experimental condition. Within each participant's list, all target pictures and distractor words appeared four times in total, once per each experimental condition. The phrasal structure condition in which each picture-word pair was first presented (same phrase vs. different phrase) was alternated across participants, meaning that possible repetition effects were not a concern. Across all participants, there was a total 12960 experimental observations, consisting of 1584 observations per each of the four experimental conditions for the young adults $(N=44)$, and 1656 observations per experimental condition for the older adults $(N=46)$, in line with Brysbaert and Stevens' (2018) recommendation for conducting a well-powered reaction time experiment (see also Simmons et al., 2011).

Procedure

Each participant was sat in a quiet testing room, facing a 22-inch monitor, wearing an OnvianTech microphone connected to a Cedrus voicekey that recorded their speech onset latencies. Audio responses were recorded by a Sony digital voice recorder. Figure 1B illustrates the sequence of stimuli presentation and timings per trial. On the experimental trials, at the offset of the fixation cross, the picture stimuli and word stimuli were presented in the centre of the screen. As soon as the stimuli appeared on screen, one or both of the stimuli began to move in a smooth motion either in the horizontal or vertical plane. The movement covered 80 pixels $(2.6 \mathrm{~cm})$ and was completed in $400 \mathrm{~ms}$. Prior to beginning the task, participants were instructed on which sentence types to use to describe the different stimuli movements. If the picture and word stimuli moved simultaneously in a horizontal plane, they were to produce a sentence with a coordinate initial phrase using the picture name first (which they would need to name independently) and the written word second (e.g., "The watch and the clock move apart"). If the word stimuli remained stationary and the picture 
stimuli moved in the vertical plane, they were to produce a sentence with a simple initial noun phrase (e.g., "The watch moves above the clock"). Participants were instructed to begin their sentence as soon as possible after the stimuli presentation for each trial. The target picture always appeared on the left of the screen in the experimental trials, meaning it was always named first.

To begin, there were 48 practice trials; the sentence types resembled those in the experimental trials (two nouns within the same phrase or within different phrases) and filler trials (singular noun phrases). The practices featured all target pictures and distractor words once (to increase participants' familiarity with the stimuli), but, critically, the practice trials did not feature any of the experimental related and unrelated picture-word pairs. If, during the practices, the participant made a lexical error (i.e., used the incorrect picture name) or syntactic error (i.e., used the wrong sentence type), they were corrected by the experimenter. The large number of practices ensured that, before beginning the experimental trials, participants were highly familiar with what sentence descriptions to produce for the different stimuli movements.

The task then continued until all four experimental blocks had been completed (consisted of 144 experimental items and 120 filler items per participant). The experimenter listened from outside the room (via a video intercom system) and noted any errors made by the participant, including incorrect picture naming (e.g., 'horn' instead of 'trumpet'), use of a different structure (e.g., "The watch moves up and the clock stays still" instead of "The watch moves above the clock"), and disfluencies, such as unnatural pauses, false starts and non-lexical fillers (e.g., "uh", "um"). ${ }^{4}$

\footnotetext{
${ }^{4}$ All participants also completed a stop-signal task and a coding task, designed to provide an indicator of their inhibitory control and processing speed respectively. Extensive details about these measurements and their analyses are available online in the 'Supplementary Materials' section of the OSF repository (https://osf.io/rwav9/).
} 


\section{Data Preparation and Analyses}

All 12960 experimental trials were included in the error analyses. For the onset latency analyses, we removed trials that contained an error, excluding $329(5.2 \%)$ young and 218 (3.3\%) older adult responses. Following Ratcliff (1993), we further excluded responses for which the onset latency was more than 2SD above/below the mean per experimental condition per age group (discarding $310(5.2 \%)$ young and $360(5.6 \%)$ older adult trials). The complete datasets used in the analyses are available online: https://osf.io/rwav9/.

All data were analysed in R (R Core Team, 2015) using generalised linear mixedeffects models (lme4 package; Bates et al., 2014). We fitted a binomial distribution to the error data as the dependent variable was categorical $($ correct $=0$; incorrect $=1)$. Following Lo and Andrews' (2015) recommendation for analysing continuous speed data, we fitted an inverse Gaussian distribution to the onset latencies with an 'identity link' function (this explicitly defines that there is a direct relationship between the predictors and the observed transformation while still satisfying the normality assumptions of the model. ${ }^{5}$ Common transformation approaches typically applied to reaction time data (i.e., logarithmic or zscores) are problematic because they can modulate the presence of interactive effects (Balota et al., 2013; Lo \& Andrews, 2015). Non-transformed data analyses that instead involve an

\footnotetext{
${ }^{5}$ We also performed a goodness-of-fit test using the 'ig_test' function in the goft package (GonzálezEstrada \& Villaseñor, 2018). In this test, the alternative hypothesis is that the distribution of the data does not follow an Inverse Gaussian distribution. Our goodness-of-fit test on the onset latencies produced a relatively high $p$ value in support of the null hypothesis $(p=.758)$, indicating that the Inverse Gaussian distribution is a plausible fit to our onset latency data.
} 
inverse Gaussian or Gamma distribution with an 'identity link' function are becoming more common in ageing research in order to overcome this issue (e.g., DeCaro \& Thomas, 2020; Smith et al., 2020).

We entered age group, semantic relatedness, and phrasal structure as fixed effects (all contrast coded as -0.5 vs. 0.5$)$. We included random intercepts for participants and items, as well as by-participant and by-item random slopes appropriate for the design. When a model did not converge with the maximal random effects structure, we simplified the random slopes, removing interactions before main effects in the order of least variance explained (as determined by the smallest variance value of the random slopes), until convergence was reached (as recommended by Barr et al., 2013; Jaeger, 2008). Lastly, in order to quantify the observed effects, we calculated Cohen's $d$ effect sizes using a method appropriate for linear mixed effect models (Brysbaert \& Stevens, 2018; Judd et al., 2017). ${ }^{6}$ We interpreted the relative sizes of the calculated effect sizes in accordance with Cohen's (1988) proposed guidelines.

\section{Results}

\section{Onset Latencies}

Figure 2 summarises young and older adults' onset latencies across the experimental conditions. Table 1 reports the best-fitting model of the onset latency data.

As expected, older adults were significantly slower than young adults (1084ms vs. 950ms, $p<.001)$. There was a main effect of phrasal structure $(p<.001)$ : participants initiated sentences slower when they began with a larger coordinate initial noun phrase (same phrase condition, $1042 \mathrm{~ms}$ ), compared to when they began with a simple initial noun phrase

\footnotetext{
${ }^{6}$ Effect size equation: Cohen's $d=\frac{\text { difference between the means }}{\sqrt{\text { var_inter }^{\text {subj }}+\text { var_inter }^{\text {item }}+\text { var_slope }^{\text {subj }}+\text { var_slope }^{\text {item }}+\text { varresidual }^{\text {ing }}}}$
} 
(different phrase condition, 996ms), indicating an overall phrasal planning scope effect of 46ms (Cohen's $d=0.66$ [medium-large]). The interaction between phrasal structure and age group was not significant $(p=.442)$, indicating that the phrasal planning scope effect was similar in young adults (47ms, Cohen's $d=0.67$ [medium-large]) and older adults (44ms, Cohen's $d=0.63$ [medium-large]).

We also observed a main effect of semantic relatedness $(p<.001)$ : participants initiated sentences slower when the target picture and distractor word were semantically related $(1033 \mathrm{~ms})$, compared to when they were semantically unrelated (1005ms), indicating an overall semantic interference effect of $28 \mathrm{~ms}$ (Cohen's $d=0.40$ [medium]). Furthermore, there was a significant interaction between semantic relatedness and age group $(p=.020)$, such that the semantic interference effect was greater for the older adults ( $34 \mathrm{~ms}$, Cohen's $d=$ 0.49 [medium]), compared to the young adults ( $21 \mathrm{~ms}$, Cohen's $d=0.30$ [small-medium]). This provides experimental evidence that semantic interference in sentence production is affected by healthy ageing.

Lastly, there was a significant interaction between semantic relatedness and phrasal structure $(p=.025)$ : overall, participants displayed larger semantic interference effects when the target picture and distractor word fell within different phrases (36ms, Cohen's $d=0.52$ [medium]), compared to within the same phrase (20ms, Cohen's $d=0.29$ [small-medium]). However, the three-way interaction between semantic relatedness, phrasal structure and age group was not significant $(p=.511)$. This indicates that, while the size of the semantic interference effect differed overall depending on whether the target picture and distractor word fell within the same phrase or in different phrases, this did not differ significantly between age groups. 


\section{Error Rates}

Figure 3 summarises young and older adults' error rates across the experimental conditions. Table 2 reports the best-fitting model of the error data.

Overall, participants' error rates were close to floor $(M=4.2 \%, S E=0.18 \%)$,

indicating that they generally performed very well on the task. Nonetheless, the analyses did reveal a main effect of semantic relatedness $(p=.016)$ : in line with the latency effects, participants produced more errors when the target picture and distractor word were semantically related, compared to when they were unrelated (4.6\% vs. $3.9 \%)$. There was also a main effect of age group $(p=.002)$, such that young adults produced more errors than older adults (5.2\% vs. 3.3\%). The direction of this effect is somewhat surprising and may be attributable to age-related increases in task motivation, leading to older adults being more engaged with lab-based tasks (Frank et al., 2015; Jackson \& Balota, 2012).

In order to investigate the possibility of a speed-accuracy trade-off in older adults, we calculated the inverse efficiency score (IES) per participant per condition (Townsend \& Ashby, 1978). ${ }^{7}$ This is a linear integration measure of each participant's onset latencies and error rates, and can be considered as the onset latency corrected for the amount of errors committed (Vandierendonck, 2017, 2018), meaning that it is able to account for possible speed-accuracy trade-off effects (for similar approaches in ageing research, see Anzures et al., 2010; Statsenko et al., 2020). Analyses of the IES using mixed-effects models produced the same effects that were observed in the onset latency analyses. This indicates that this

377 positive age effect in the error rates was not the result of a speed-accuracy trade-off and that 378 it did not influence the observed onset latency effects.

\footnotetext{
${ }^{7}$ IES $=$ average onset latency $/(1-$ proportion of errors $)$
} 
[Figure 3 about here]

[Table 2 about here]

382

\section{Discussion}

We investigated the effect of healthy ageing on the syntactic and lexical processes involved in sentence production using a picture-word semantic interference task. We used speech onset latencies as this provides a reliable index of underlying sentence planning and semantic interference processes (Bürki et al., 2020; Glaser \& Düngelhoff, 1984; Martin et al., 2010, 2014; Meyer, 1996; Smith \& Wheeldon, 1999, 2004). Our main findings are three-fold. Firstly, young and older adults initiated sentences slower when they contained larger, compared to smaller, initial phrases, indicating that both age groups were engaging in a phrasal scope of advanced planning. Secondly, the magnitude of the semantic interference effect was larger for older adults than for young adults, indicating an age-related increase in lexical competition. Thirdly, for young and older adults, the magnitude of the semantic interference effect was larger when the target picture and distractor word fell within different phrases. This indicates that both age groups experienced greater interference when the distractor word (which they would have automatically read and processed prior to beginning articulation) was not contained within the initial to-be-produced phrase. Together, our findings provide evidence that lexical competition during sentence production increases with age, but that syntactic planning skills are preserved. Moreover, young and older adults are similarly affected by the syntactic relationship between competing lexical items.

To first consider the evidence of preserved phrasal planning scope, our finding that speakers are slower to begin sentences with larger initial phrases is consistent with previous studies that have similarly used onset latency measures to specifically target incremental sentence planning processes in young adults (Martin et al., 2010, 2014; Smith \& Wheeldon, 
1999) and in older adults (Hardy et al., 2020). Speech onset latencies are indicative of the amount of pre-planning required prior to articulation (slower speech onset latencies indicating more planning) and are therefore informative about underlying linguistic processes that must also occur during more naturalistic speech production (Levelt, 1989; Wheeldon, 2013). Within this current study, we manipulated the length and complexity of the initial phrase structure (coordinate vs. simple noun phrase) while keeping lexical factors equal across the two phrase conditions. Thus, the slower onset latencies observed for the lengthier initial phrase condition can be attributed to differences in the time required for the planning of the first syntactic phrasal unit (which requires both syntax generation and lexical retrieval).

We found similar effects in both age groups, which indicates that both young and older adults were engaged in a phrasal scope of advanced planning, such that they prioritised the generation of syntax and lexical retrieval within the first phrase prior to articulation and planned incrementally in phrasal units. Importantly, we replicated the findings of Hardy et al. (2020) using a different task, involving different sentence structures and lexical items (see also Spieler \& Griffin, 2006). Together, these studies provide robust evidence that, despite age-related declines in other cognitive domains, syntactic planning scope is preserved with age and that older adults engage in a phrasal scope of advanced planning when producing sentences. This finding fits with Peelle's (2019) 'supply and demand' framework, which suggests that behavioural success reflects a complex balance between task requirements and the level/type of cognitive resources available to the speaker (see Ferré et al., 2020, for another example of the application of this framework to age effects on language production). In the case of syntactic processing during the production of sentences with different initial phrase structures, older speakers maintain sufficient cognitive capacity to plan in the same way as young adults. However, when the processing load was increased by the introduction of a semantic interference component, age-related differences did emerge. 
Both young and older adults displayed semantic interference, in that they were slower

to initiate sentences when the target picture and distractor word were semantically related

Meyer, 1996; Smith \& Wheeldon, 2004). This is because the rapid and automatic processing of the written distractor word prior to the lexical retrieval of the target picture name resulted in increased lexical competition when the two were semantically related. This lexical competition may have arisen because of the co-activation of lemma information of the target picture and semantically related concepts, which then compete for selection (Levelt et al., 1999; Roelofs, 1992, 1997), or reflect the speed with which the distractor word can be excluded as a potential articulatory response to the target picture (Finkbeiner \& Caramazza, 2006; Mahon et al., 2007). Our study was not specifically designed to distinguish between different theoretical accounts of semantic interference and both accounts may offer a valid explanation of our finding. Critically though, the semantic interference effect was larger for older speakers, compared to young speakers. Although the average group difference was small in magnitude, this effect was statistically significant and further supported by age group differences in the Cohen's $d$ effect size (larger for older adults), indicating a meaningful agerelated effect. Our task was designed to tap into how speakers manage the temporal coactivation of competing lexical items during sentence planning and therefore provides the first evidence that lexical competition increases with age at the sentence level. Moreover, by examining semantic interference in sentence production (as opposed to single word production), our study provides evidence of lexical competition in a context which is more akin to everyday language production, albeit still within a constrained experimental task and naturalistic settings; Vitevitch, 2002). 
Our finding of an age group effect is consistent with some studies of single word production that have also found age-related increases in lexical competition (Britt et al., 2016; Taylor \& Burke, 2002), but not other studies that have found no age differences (Gordon \& Cheimariou, 2013; Mulatti et al., 2014). We suggest that these previous mixed findings occurred because producing a single word is often insufficiently challenging to outweigh older speakers' cognitive resources for a given task (Peelle, 2019). Much more processing, however, is required to produce a multi-word sentence. In particular, when words form constituent parts of a larger sentence structure, there is a temporal overlap of linguistic information between different lexical items within the structure (i.e., horizontal flow; Rapp \& Samuel, 2002; Smith \& Wheeldon, 2004). This can lead to greater processing demands to resolve any lexical competition since the associations between words at the sentence level are more complex than those between single words (Sass et al., 2010). Indeed, while Belke and Meyer (2007) did not find age differences in semantic interference during single word processing, differences did emerge when participants named multiple objects as part of a list. Together with our finding, this indicates that age-related differences in lexical competition do exist during speech production, but that this may only become apparent during the production of multi-word utterances in which there is a more complex flow of linguistic information between lexical items. Although our task was an experimental one, we would expect our findings to generalise to other more naturalistic speech production contexts in line with studies that have found similar ageing effects on language production in controlled and naturalistic settings (Burke et al., 1991; Rabaglia \& Salthouse, 2011).

We now turn to our findings of the effect of phrasal structure on semantic interference. We found that speakers displayed the greatest interference (i.e., slowed onset latencies) when the related target picture and distractor word fell within different phrases, compared to within the same phrase, and critically that this did not vary between age groups. 
479 The overall direction of this effect may at first seem surprising given that lexical items within

480

481

482

483 the same phrase may be considered more closely connected and, therefore, more likely to be in competition. However, a valid explanation does exist when the characteristics of the picture-word interference paradigm and the multi-composited nature of sentence planning are considered. Specifically, in our task, participants would have accessed lexical information relating to the distractor word as soon as it appeared on screen and prior to beginning speech planning because reading is a fast and highly automated process that cannot be prevented in skilled readers (Augustinova \& Ferrand, 2014; LaBerge \& Samuels, 1974; Samuels \& Flor, 1997). Regardless of the distractor's position in the to-be-produced sentence, participants would then have sought to plan the initial phrase prior to articulation (as evidenced by participants' increased time taken to initiate sentences with larger initial phrases; see also Hardy et al., 2020; Martin et al., 2010, 2014). Speakers did not have a choice about which syntactic structure to produce as they were instructed to produce a specific sentence type for a specific stimuli movement. This meant that they had to deal with the early activation of lexical information relating to the distractor word that was either required in the initial phrase or not required until later in the sentence (appeared within the second phrase).

When the target picture and semantically related distractor word both fell within the initial phrase (same phrase condition), the presentation of the distractor created semantic interference in the retrieval of the target picture name (i.e., increased lexical load), but not in the pre-articulatory syntactic planning because the distractor word was also included in the planning of the initial phrasal unit. By contrast, when only the target picture fell within the initial phrase, and the semantic distractor word was in the second phrase (different phrase condition), in addition to interfering with the retrieval of the target picture name, the information relating to the distractor word would have also interfered with the syntactic planning of the initial phrasal unit in which it did not feature, thereby placing greater 
504

505

506

507

508

509

510

511

512

513

514

515

516

517

518

519

520

521

522

523

524

525

526

527

528

demands on the cognitive resources involved in maintaining linearisation of output. Thus, in the different phrase condition, the premature access to lexical information meant that speakers had to resolve interference from the distractor word at both the lexical and syntactic level, leading to an additive disruptive effect on sentence planning prior to articulation. These differences in where prematurely-accessed lexical information can be incorporated into a tobe-produced sentence (first or second phrase) can therefore explain why we observed a greater slowing of participants' speech onset latencies dependent on whether the target picture and distractor word fell within the same phrase or different phrases. Our findings fit with existing theoretical models of the automaticity of reading (LaBerge \& Samuels, 1974), as well as with models of speech production involving an incremental system of planning meaning that different processing components can be simultaneously activated (e.g., Bock \& Levelt, 1994; Levelt et al., 1999). Moreover, our findings contribute towards the wider understanding of the effect of lexical availability on sentence planning as we demonstrate that early access to upcoming lexical items is not beneficial to the speed of speech production when the premature access elicits lexical competition and interferes with pre-articulatory syntactic planning. Premature lexical access, such as in the form of a picture preview, does elicit some benefits though when the previewed lexical item is not semantically related to other lexical items in the sentence as this increased lexical availability can aid the planning and production of the initial phrase (Hardy et al., 2020; Wheeldon et al., 2013). Together, these findings demonstrate that the speed of sentence planning depends upon a complex interplay between the lexical properties of different words, as well as their syntactic position within the to-be-produced sentence.

Notably, while we observed that the magnitude of the semantic interference effect varied across phrasal structure conditions, this did not differ between age groups. This indicates that despite an age-related increase in lexical competition (as evidenced by an 
overall age group difference in semantic interference), older adults were not disadvantaged by the competing lexical items being in different phrases to any greater extent than the young adults were. One explanation for this is that whether the target picture and distractor word were in the same or different phrases tapped into syntactic processing during sentence production, which may be more preserved with age than lexical processing (Hardy et al., 2020). As we suggested previously, the difference in the magnitude of the semantic interference effect between the same phrase and different phrase conditions was not driven by increases in lexical competition between the two related lexical items, but instead resulted from increased disruption at the syntactic planning level because the distractor word was not part of the initial phrasal unit. This may explain why we did not observe any age group differences since only the syntactic load changed depending on whether the target picture and distractor word were in the same phrase or different phrases, whereas the lexical processing load remained the same (i.e., the semantically related distractor interfered with the retrieval of the target picture name to the same extent in both conditions). Our findings therefore provide further evidence for the divergent effect of old age on lexical and syntactic processing during sentence production (Hardy et al., 2020), and add to the growing evidence that healthy ageing does not affect all aspects of language equally (Burke \& Shafto, 2008; Peelle, 2019).

In summary, our study investigated young and older adults' semantic interference during sentence production in order to provide novel insight into age-related effects on lexical and syntactic on-line processing. Firstly, our study provides evidence of age-related disruption to the processes involved in managing the temporal flow of lexical information during sentence production, such that older adults experienced greater interference when two lexical items in a to-be-produced sentence were semantically related. In contrast, we found evidence of an age-related preservation of syntactic processing. Both age groups engaged in a phrasal scope of advanced planning and were similarly affected by the syntactic relationship 
between competing lexical items (i.e., whether they were in the same phrase or different phrases). Although we employed fairly simple syntactic structures within the current study, we would expect our findings to generalise to sentence production more widely given the

557 universality of planning scope and semantic interference effects across different sentence 558 types (e.g., Allum \& Wheeldon, 2007; Ferreira, 1991; Meyer, 1996; Momma et al., 2016).

559 Importantly, within a constrained experimental task, we observed evidence of age-related 560 decline and preservation that are attributable to linguistic processes that must also form a part

561 of more naturalistic language processing. We do though emphasise the importance of further 562 research to identify the extent to which these findings generalise to other less-constrained 563 speaking contexts and syntactic constructions. Overall, our findings underscore that there is 564 not a straightforward relationship between language and ageing, and highlight how each 565 aspect of language processing must be carefully considered on an individual basis when investigating how it is affected by healthy ageing. 


\section{Author Notes}

Acknowledgments. We gratefully acknowledge the help of Eva Staff with data collection, and Denise Clissett with participant recruitment.

Funding details. This research was supported by an Economic and Social Research Council (ESRC) studentship awarded to Sophie M. Hardy from the University of Birmingham Doctoral Training Centre (grant number: ES/J50001X/1).

Disclosure statement. The authors report no potential conflict of interest.

Data availability. The complete datasets, stimuli list and supplementary materials of the study are provided online in a dedicated repository on the Open Science Framework (https://osf.io/rwav9/). 


\section{Table 1}

Summary of the best-fitting model of the onset latency data.

\begin{tabular}{lcccc}
\hline Predictor & Coefficient & SE & $\boldsymbol{t}$-value & $\boldsymbol{p}$ \\
\hline A: All data & & & & \\
\hline Intercept & 1119.34 & 7.16 & 156.42 & $<.001$ \\
Semantic Relatedness & 28.06 & 5.45 & 5.15 & $<.001$ \\
Phrasal Structure & 51.31 & 4.22 & 12.16 & $<.001$ \\
Age Group & -118.86 & 11.97 & -9.93 & $<.001$ \\
Semantic Relatedness * Phrasal Structure & -14.04 & 6.28 & -2.24 & .025 \\
Relatedness * Age Group & -14.70 & 6.30 & -2.33 & .020 \\
Phrasal Structure * Age Group & 6.48 & 8.44 & 0.77 & .442 \\
Semantic Relatedness * Phrasal Structure & & & & .511 \\
* Age Group & 8.25 & 12.56 & 0.66 & \\
\hline
\end{tabular}

Note. The model converged with random intercepts for participants and items with additional byparticipant random slopes for the main effects of semantic relatedness and phrasal structure, and a byitem random slope for the main effect of semantic relatedness. 


\section{Table 2}

Summary of the best-fitting model of the error data.

\begin{tabular}{lcccc}
\hline Predictor & Coefficient & SE & Wald $Z$ & $\boldsymbol{p}$ \\
\hline Intercept & -3.68 & 0.14 & -26.02 & $<.001$ \\
Semantic Relatedness & 0.30 & 0.12 & 2.42 & .016 \\
Phrasal Structure & 0.26 & 0.15 & 1.68 & .094 \\
Age Group & 0.66 & 0.22 & 3.02 & .002 \\
Semantic Relatedness * Phrasal Structure & -0.10 & 0.24 & -0.42 & .674 \\
Semantic Relatedness * Age Group & -0.18 & 0.20 & -0.89 & .373 \\
Phrasal Structure * Age Group & 0.41 & 0.21 & 1.93 & .054 \\
Semantic Relatedness * Phrasal Structure & & & & \\
$*$ Age Group & 0.24 & 0.39 & 0.61 & .541 \\
\hline
\end{tabular}

Note. The model converged with random intercepts for participants and items with additional byparticipant random slopes for the main effects and interactions of semantic relatedness and phrasal structure, and a by-item random slope for the main effect of phrasal structure. 


\section{Figure Captions}

Figure 1. Picture-word interference sentence production task design (A) and trial events (B). The target picture and the distractor word appeared simultaneously and aligned centrally in the horizontal plane (the picture was always on the left). The movement of the appropriate stimuli began immediately in a smooth motion and was completed in 400ms (the arrows in the figure pictorially depict the actual movement). Speech onset latencies were recorded from the onset of the stimuli to when the participant began to speak. The picture and written word disappeared 1000ms after the participant finished speaking (i.e., had completed their picture description) or $4000 \mathrm{~ms}$ after the onset of the stimuli if the participant did not provide a response.

Figure 2. Onset latencies for young and older adults when producing sentences that contained semantically related or unrelated lexical items that either fell within the same phrase or within different phrases. Box-and-whisker plots and violin spreads represent the distribution of the data (figure code modified from Allen et al., 2019). The diamonds denote the mean per condition, above which is written the mean value (in bold) and the standard error (in italics in brackets).

Figure 3. Error rates for young and older adults when producing sentences that contained semantically related or unrelated lexical items that either fell within the same phrase or within different phrases. The diamonds denote the mean per condition (values written below); boxand-whisker plots represent the distribution of the error rates across participants. 
Figure 1

A

PHRASAL STRUCTURE

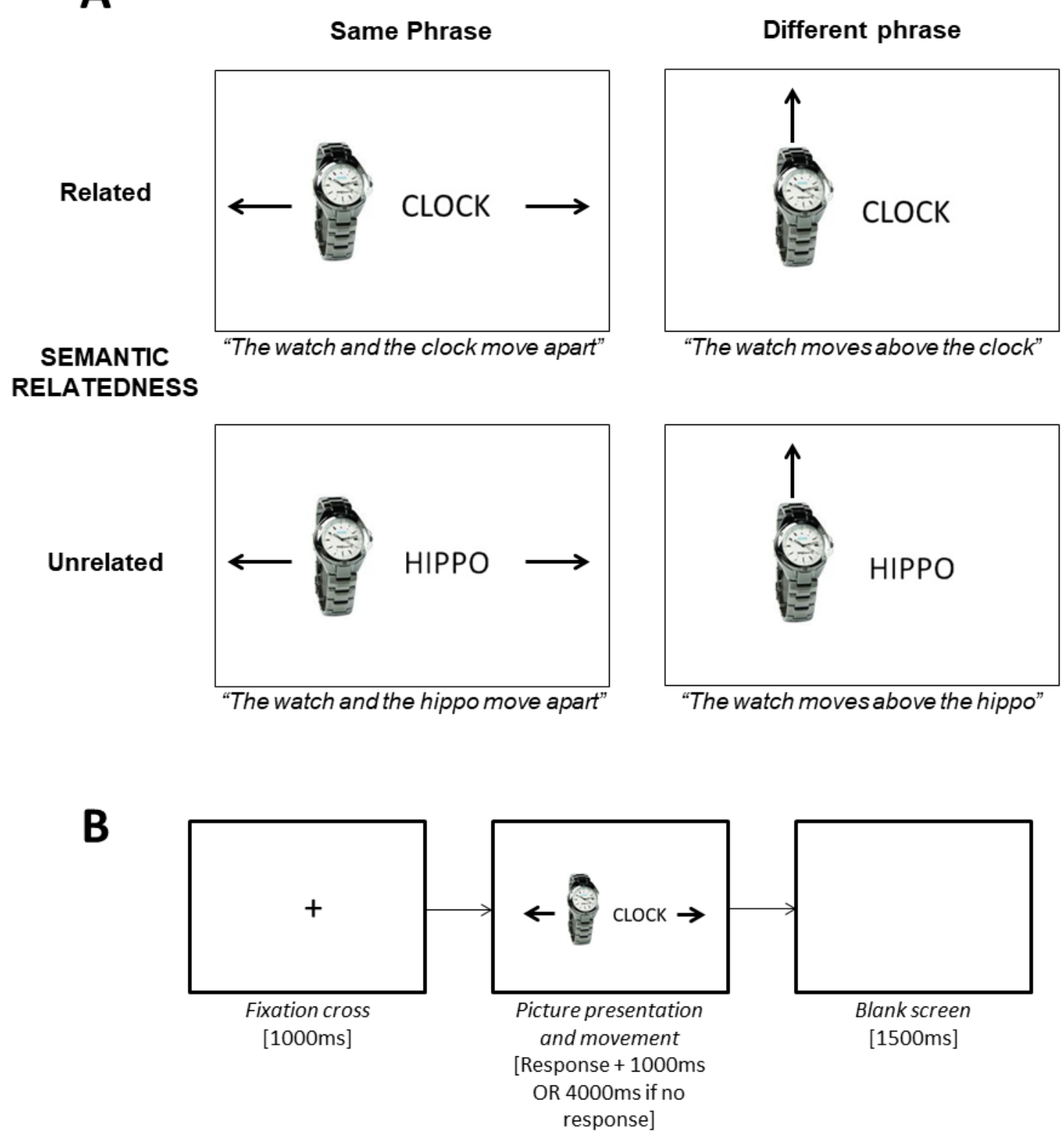


Figure 2

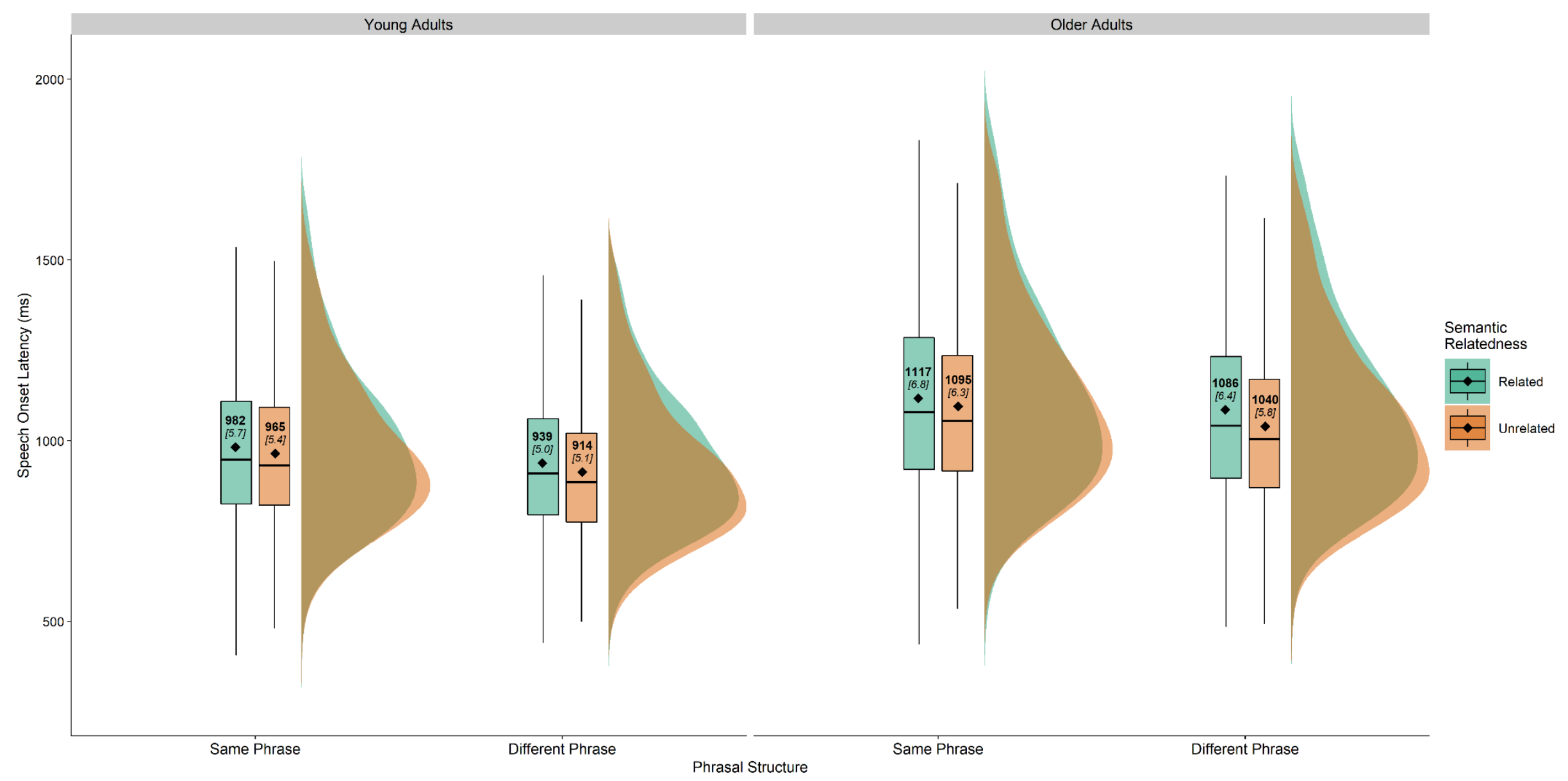


Figure 3

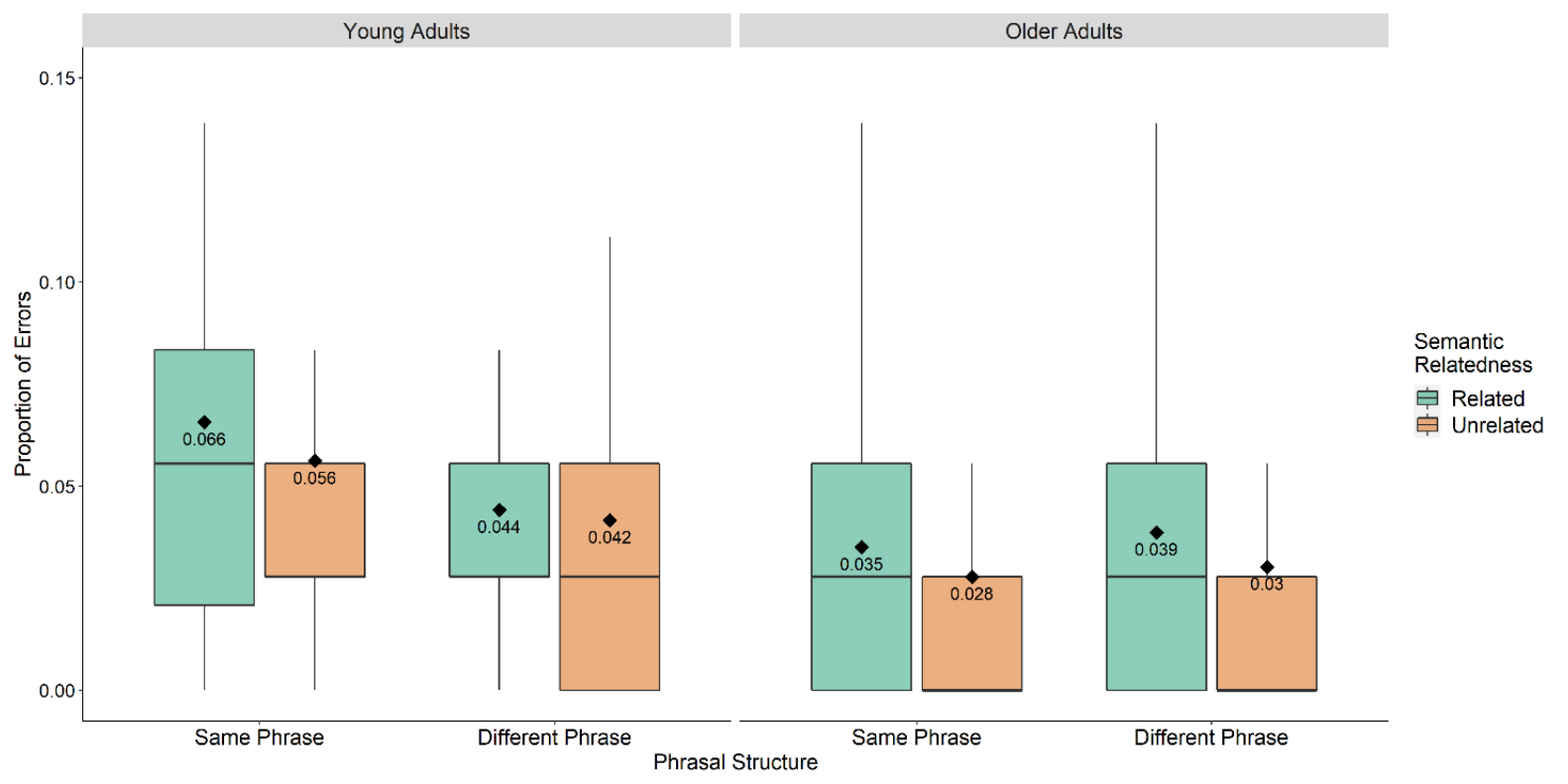




\section{References}

Allen, M., Poggiali, D., Whitaker, K., Marshall, T. R., \& Kievit, R. A. (2019). Raincloud plots: A multi-platform tool for robust data visualization. Wellcome Open Research, 4(63). https://doi.org/10.12688/wellcomeopenres.15191.1

Allum, P. H., \& Wheeldon, L. (2007). Planning scope in spoken sentence production: The role of grammatical units. Journal of Experimental Psychology: Learning, Memory, and Cognition, 33(4), 791-810. https://doi.org/10.1037/0278-7393.33.4.791

Altmann, L. J. P., \& Kemper, S. (2006). Effects of age, animacy and activation order on sentence production. Language and Cognitive Processes, 21(1-3), 322-354. https://doi.org/10.1080/0169096054400006

Anzures, G., Ge, L., Wang, Z., Itakura, S., \& Lee, K. (2010). Culture shapes efficiency of facial age judgments. PLoS ONE, 5(7). https://doi.org/10.1371/journal.pone.0011679

Augustinova, M., \& Ferrand, L. (2014). Automaticity of word reading: Evidence From the semantic stroop paradigm. Current Directions in Psychological Science, 23(5), 343348. https://doi.org/10.1177/0963721414540169

Balota, D. A., Aschenbrenner, A. J., \& Yap, M. J. (2013). Additive effects of word frequency and stimulus quality: The influence of trial history and data transformations. Journal of Experimental Psychology: Learning Memory and Cognition, 39(5), 1563-1571. https://doi.org/10.1037/a0032186

Barr, D. J., Levy, R., Scheepers, C., \& Tily, H. J. (2013). Random effects structure for confirmatory hypothesis testing: Keep it maximal. Journal of Memory and Language, 68(3), 255-278. https://doi.org/10.1016/j.jml.2012.11.001

Bates, D., Mächler, M., Bolker, B. M., \& Walker, S. C. (2014). Fitting linear mixed-effects models using lme4. Journal of Statistical Software, 67(1), 1-48. https://doi.org/10.18637/jss.v067.i01

Belke, E., \& Meyer, A. S. (2007). Single and multiple object naming in healthy ageing. Language and Cognitive Processes, 22(8), 1178-1211. https://doi.org/10.1080/01690960701461541

Bock, K., \& Levelt, W. J. M. (1994). Language production: Grammatical encoding. In M. A. Gernsbacher (Ed.), Handbook of psycholinguistics (pp. 945-984). Academic Press.

Bopp, K. L., \& Verhaeghen, P. (2005). Aging and verbal memory span: A meta-analysis. Journals of Gerontology: Psychological Sciences, 60B(5), 223-233. https://doi.org/10.1093/geronb/60.5.P223 
Britt, A. E., Ferrara, C., \& Mirman, D. (2016). Distinct effects of lexical and semantic competition during picture naming in younger adults, older adults, and people with aphasia. Frontiers in Psychology, 7, 813. https://doi.org/10.3389/fpsyg.2016.00813

Brysbaert, M., \& Stevens, M. (2018). Power analysis and effect size in mixed effects models: A Tutorial. Journal of Cognition, 1(1), 9. https://doi.org/10.5334/joc.10

Burke, D. M. (2002). Language production and aging. In S. Kemper \& R. Kliegl (Eds.), Constraints on language: Aging, grammar, and memory (pp. 3-27). Kluwer Academic Publishers.

Burke, D. M., MacKay, D. G., Worthley, J. S., \& Wade, E. (1991). On the tip of the tongue: What causes word-finding failures in young and older adults. Journal of Memory and Language, 30, 542-579. https://doi.org/10.1016/0749-596X(91)90026-G

Burke, D. M., \& Shafto, M. A. (2008). Language and aging. In F. I. M. Craik \& T. A. Salthouse (Eds.), The handbook of aging and cognition (pp. 373-443). Psychology Press. https://doi.org/10.1080/03610738508259280

Bürki, A., Elbuy, S., Madec, S., \& Vasishth, S. (2020). What did we learn from forty years of research on semantic interference? A Bayesian meta-analysis. Journal of Memory and Language, 114, 104125. https://doi.org/10.1016/j.jml.2020.104125

Cohen, J. (1988). Statistical power analysis for the behavioral sciences (2nd ed). L. Erlbaum Associates.

Davidson, D. J., Zacks, R. T., \& Ferreira, F. (2003). Age preservation of the syntactic processor in production. Journal of Psycholinguistic Research, 32(5), 541-566. https://doi.org/10.1023/A:1025402517111

DeCaro, R., \& Thomas, A. K. (2020). Prompting retrieval during monitoring and selfregulated learning in older and younger adults. Metacognition and Learning, 15(3), 367-390. https://doi.org/10.1007/s11409-020-09230-y

Dell, G. S. (1986). A spreading-activation theory of retrieval in sentence production. Psychological Review, 93(3), 283-321. https://doi.org/10.1037/0033-295X.93.3.283

Ferré, P., Jarret, J., Brambati, S. M., Bellec, P., \& Joanette, Y. (2020). Task-Induced Functional Connectivity of Picture Naming in Healthy Aging: The Impacts of Age and Task Complexity. Neurobiology of Language, 1(2), 161-184. https://doi.org/10.1162/nol_a_00007

Ferreira, F. (1991). Effects of length and syntactic complexity on initiation times for prepared utterances. Journal of Memory and Language, 30(2), 210-233. https://doi.org/10.1016/0749-596X(91)90004-4 
Ferreira, F., \& Swets, B. (2002). How incremental is language production? Evidence from the production of utterances requiring the computation of arithmetic sums. Journal of Memory and Language, 46(1), 57-84. https://doi.org/10.1006/jmla.2001.2797

Finkbeiner, M., \& Caramazza, A. (2006). Lexical selection is not a competitive process: A reply to La Heij et al. (2006). Cortex, 42(7), 1032-1036. https://doi.org/10.1016/S0010-9452(08)70210-7

Frank, D. J., Nara, B., Zavagnin, M., Touron, D. R., \& Kane, M. J. (2015). Validating older adults' reports of less mind-wandering: An examination of eye movements and dispositional influences. Psychology and Aging, 30(2), 266-278. https://doi.org/10.1037/pag0000031

Glaser, W. R., \& Düngelhoff, F. J. (1984). The time course of picture-word interference. Journal of Experimental Psychology: Human Perception and Performance, 10(5), 640-654. https://doi.org/10.1037/0096-1523.10.5.640

González-Estrada, E., \& Villaseñor, J. A. (2018). An R package for testing goodness of fit: Goft. Journal of Statistical Computation and Simulation, 88(4), 726-751. https://doi.org/10.1080/00949655.2017.1404604

Gordon, J. K., \& Cheimariou, S. (2013). Semantic interference in a randomized naming task: Effects of age, order, and category. Cognitive Neuropsychology, 30(7-8), 476-494. https://doi.org/10.1080/02643294.2013.877437

Hardy, S. M., Messenger, K., \& Maylor, E. A. (2017). Aging and syntactic representations: Evidence of preserved syntactic priming and lexical boost. Psychology and Aging, 32(6), 588-596. https://doi.org/10.1037/pag0000180

Hardy, S. M., Segaert, K., \& Wheeldon, L. (2020). Healthy ageing and sentence production: Disrupted lexical access in the context of intact syntactic planning. Frontiers in Psychology, 11, 257. https://doi.org/10.3389/fpsyg.2020.00257

Jackson, J. D., \& Balota, D. A. (2012). Mind-wandering in Younger and Older Adults: Converging Evidence from the Sustained Attention to Response Task and Reading for Comprehension. Psychology and Aging, 27(1), 106-119. https://doi.org/10.1037/a0023933

Jaeger, T. F. (2008). Categorical data analysis: Away from ANOVAs (transformation or not) and towards logit mixed models. Journal of Memory and Language, 59(4), 434-446. https://doi.org/10.1016/j.jml.2007.11.007 
Judd, C. M., Westfall, J., \& Kenny, D. A. (2017). Experiments with more than one random factor: Designs, analytic models, and statistical power. Annual Review of Psychology, 68(1), 601-625. https://doi.org/10.1146/annurev-psych-122414-033702

Kemper, S., Greiner, L. H., Marquis, J. G., Prenovost, K., \& Mitzner, T. L. (2001). Language decline across the life span: Findings from the Nun Study. Psychology and Aging, 16(2), 227-239. https://doi.org/10.1037/0882-7974.16.2.227

Kemper, S., Herman, R. E., \& Lian, C. H. T. (2003). Age differences in sentence production. Journal of Gernotology: Psychological Sciences, 58(5), 260-269. https://doi.org/10.1093/geronb/58.5.P260

Kemper, S., Herman, R. E., \& Liu, C. J. (2004). Sentence production by young and older adults in controlled contexts. Journals of Gerontology: Series B, 58B, 220-224. https://doi.org/10.1093/geronb/59.5.P220

LaBerge, D., \& Samuels, S. J. (1974). Toward a theory of automatic information processing in reading. Cognitive Psychology, 6(2), 293-323. https://doi.org/10.1016/00100285(74)90015-2

LaGrone, S., \& Spieler, D. H. (2006). Lexical competition and phonological encoding in young and older speakers. Psychology and Aging, 21(4), 804-809. https://doi.org/10.1037/0882-7974.21.4.804

Levelt, W. J. M. (1989). Speaking: From intention to articulation. MIT Press.

Levelt, W. J. M., \& Maassen, B. (1981). Lexical search and order of mention in sentence production. In W. J. M. Levelt \& W. Klein (Eds.), Crossing the boundaries in linguistics (pp. 221-252). D. Reidel Publishing Company.

Levelt, W. J. M., Roelofs, A., \& Meyer, A. S. (1999). A theory of lexical access in speech production. Behavioral and Brain Sciences, 22, 1-75. https://doi.org/10.1017/S0140525X99001776

Lo, S., \& Andrews, S. (2015). To transform or not to transform: Using generalized linear mixed models to analyse reaction time data. Frontiers in Psychology, 6, 1171. https://doi.org/10.3389/fpsyg.2015.01171

Mahon, B. Z., Costa, A., Peterson, R., Vargas, K. A., \& Caramazza, A. (2007). Lexical selection is not by competition: A reinterpretation of semantic interference and facilitation effects in the picture-word interference paradigm. Journal of Experimental Psychology: Learning, Memory, and Cognition, 33(3), 503-535. https://doi.org/10.1037/0278-7393.33.3.503 
Martin, R. C., Crowther, J. E., Knight, M., Tamborello, F. P., \& Yang, C. L. (2010). Planning in sentence production: Evidence for the phrase as a default planning scope. Cognition, 116(2), 177-192. https://doi.org/10.1016/j.cognition.2010.04.010

Martin, R. C., Yan, H., \& Schnur, T. T. (2014). Working memory and planning during sentence production. Acta Psychologica, 152, 120-132. https://doi.org/10.1016/j.actpsy.2014.08.006

Meyer, A. S. (1996). Lexical access in phrase and sentence production: Results from pictureword interference experiments. Journal of Memory and Language, 35(4), 477-496. https://doi.org/10.1006/jmla.1996.0026

Momma, S., Slevc, L. R., \& Phillips, C. (2016). The timing of verb selection in Japanese sentence production. Journal of Experimental Psychology: Learning, Memory, and Cognition, 42(5), 813-824. https://doi.org/10.1037/xlm0000195

Mulatti, C., Calia, C., De Caro, M. F., \& Della Sala, S. (2014). The cumulative semantic interference effect in normal and pathological ageing. Neuropsychologia, 65, 125130. https://doi.org/10.1016/J.NEUROPSYCHOLOGIA.2014.10.007

Nasreddine, Z. S., Phillips, N. A., Bedirian, V., Charbonneau, S., Whitehead, V., Collin, I., Cummings, J. L., \& Chertkow, H. (2005). The Montreal cognitive assessment, MoCA: A brief screening for mild cognitive impairment. Journal of the American Geriatrics Society, 53, 695-699. https://doi.org/10.1111/j.1532-5415.2005.53221.x

Peelle, J. E. (2019). Language and aging. In G. I. de Zubicaray \& N. O. Schiller (Eds.), The Oxford handbook of neurolinguistics (pp. 295-216). Oxford University Press. https://doi.org/10.1016/B978-0-12-809324-5.01889-7

R Core Team. (2015). A language and environment for statistical computing. R Foundation for Statistical Computing. https://www.r-project.org/

Rabaglia, C. D., \& Salthouse, T. A. (2011). Natural and constrained language production as a function of age and cognitive abilities. Language and Cognitive Processes, 26(10), 1505-1531. https://doi.org/10.1080/01690965.2010.507489

Rapp, D. N., \& Samuel, A. G. (2002). A reason to rhyme: Phonological and semantic influences on lexical access. Journal of Experimental Psychology: Learning Memory and Cognition, 28(3), 564-571. https://doi.org/10.1037//0278-7393.28.3.564

Ratcliff, R. (1993). Methods for dealing with RT outliers. Psychological Bulletin, 114(3), 510-532. https://doi.org/10.1037/0033-2909.114.3.510

Roelofs, A. (1992). A spreading-activation theory of lemma retrieval in speaking. Cognition, 42(1-3), 107-142. https://doi.org/10.1016/0010-0277(92)90041-F 
Roelofs, A. (1997). The WEAVER model of word-form encoding in speech production. Cognition, 64(3), 249-284. https://doi.org/10.1016/S0010-0277(97)00027-9

Roelofs, A., \& Ferreira, V. S. (2019). The architecture of speaking. In P. Hagoort (Ed.), Human language: From genes and brains to behavior (pp. 35-50). MIT Press.

Samuels, S. J., \& Flor, R. F. (1997). The importance of automaticity for developing expertise in reading. Reading \& Writing Quarterly, 13(2), 107-121. https://doi.org/10.1080/1057356970130202

Sass, K., Heim, S., Sachs, O., Theede, K., Muehlhaus, J., Krach, S., \& Kircher, T. (2010). Why the leash constrains the dog: The impact of semantic associations on sentence production. Acta Neurobiologiae Experimentalis, 70(4), 435-453.

Schneider, W., Eschman, A., \& Zuccolotto, A. (2002). E-prime user's guide. Psychology Software Tools Inc.

Schriefers, H., Meyer, A. S., \& Levelt, W. J. M. (1990). Exploring the time course of lexical access in language production: Picture-word interference studies. Journal of Memory and Language, 29(1), 86-102. https://doi.org/10.1016/0749-596X(90)90011-N

Segaert, K., Lucas, S. J. E., Burley, C. V., Segaert, P., Milner, A. E., Ryan, M., \& Wheeldon, L. (2018). Higher physical fitness levels are associated with less language decline in healthy ageing. Scientific Reports, 8, 6715. https://doi.org/10.1038/s41598-01824972-1

Simmons, J. P., Nelson, L. D., \& Simonsohn, U. (2011). False-positive psychology: Undisclosed flexibility in data collection and analysis allows presenting anything as significant. Psychological Science, 22(11), 1359-1366. https://doi.org/10.1177/0956797611417632

Smith, M. E., Newberry, K. M., \& Bailey, H. R. (2020). Differential effects of knowledge and aging on the encoding and retrieval of everyday activities. Cognition, 196, 104159. https://doi.org/10.1016/j.cognition.2019.104159

Smith, M., \& Wheeldon, L. (1999). High level processing scope in spoken sentence production. Cognition, 73(3), 205-246. https://doi.org/10.1016/S00100277(99)00053-0

Smith, M., \& Wheeldon, L. (2004). Horizontal information flow in spoken sentence production. Journal of Experimental Psychology: Learning, Memory, and Cognition, 30(3), 675-686. https://doi.org/10.1037/0278-7393.30.3.675 
Smith, T., Gildeh, N., \& Holmes, C. (2007). The Montreal Cognitive Assessment: Validity and utility in a memory clinic setting. Canadian Journal of Psychiatry, 52(5), 329332. https://doi.org/10.1177/070674370705200508

Spieler, D. H., \& Griffin, Z. M. (2006). The influence of age on the time course of word preparation in multiword utterances. Language and Cognitive Processes, 21(1-3), 291-321. https://doi.org/10.1080/01690960400002133

Starreveld, P. A., \& La Heij, W. (1996). Time-course analysis of semantic and orthographic context effects in picture naming. Journal of Experimental Psychology: Learning, Memory, and Cognition, 22(4), 896-918. https://doi.org/10.1037/0278-7393.22.4.896

Statsenko, Y., Habuza, T., Gorkom, K. N.-V., Zaki, N., \& Almansoori, T. M. (2020). Applying the inverse efficiency score to visual-motor task for studying speedaccuracy performance while aging. Frontiers in Aging Neuroscience, 12. https://doi.org/10.3389/fnagi.2020.574401

Stine-Morrow, E. A. L., Miller, L. M. S., Gagne, D. D., \& Hertzog, C. (2008). Self-regulated reading in adulthood. Psychology and Aging, 23(1), 131-153. https://doi.org/10.1016/B978-0-12-811448-3.00002-4

Taylor, J. K., \& Burke, D. M. (2002). Asymmetric aging effects on semantic and phonological processes: Naming in the picture-word interference task. Psychology and Aging, 17(4), 662-676. https://doi.org/10.1037/0882-7974.17.4.662

Tipper, S. P. (1991). Less attentional selectivity as a result of declining inhibition in older adults. Bulletin of the Psychonomic Society, 29(1), 45-47. https://doi.org/10.3758/BF03334765

Townsend, J. T., \& Ashby, F. G. (1978). Methods of modeling capacity in simple processing systems. In J. N. J. Castellan \& F. Restle (Eds.), Cognitive Theory (pp. 199-239). Lawrence Erlbaum Associates.

Tree, J. J., \& Hirsh, K. W. (2003). Sometimes faster, sometimes slower: Associative and competitor priming in picture naming with young and elderly participants. Journal of Neurolinguistics, 16(6), 489-514. https://doi.org/10.1016/S0911-6044(02)00005-2

Tun, P. A., O'Kane, G., \& Wingfield, A. (2002). Distraction by competing speech in young and older adult listeners. Psychology and Aging, 17(3), 453-467. https://doi.org/10.1037//0882-7974.17.3.453

United Nations. (2011). International standard classification of education. UN Institute for Statistics. 
Vandierendonck, A. (2017). A comparison of methods to combine speed and accuracy measures of performance: A rejoinder on the binning procedure. Behavior Research Methods, 49(2), 653-673. https://doi.org/10.3758/s13428-016-0721-5

Vandierendonck, A. (2018). Further tests of the utility of integrated speed-accuracy measures in task switching. Journal of Cognition, 1(1). https://doi.org/10.5334/joc.6

Verhaeghen, P. (2003). Aging and vocabulary scores: A meta-analysis. Psychology and Aging, 18(2), 332-339. https://doi.org/10.1037/0882-7974.18.2.332

Vitevitch, M. S. (2002). Naturalistic and experimental analyses of word frequency and neighborhood density effects in slips of the ear. Language and Speech, 45(4), 407434. https://doi.org/10.1177/00238309020450040501

Wagner, V., Jescheniak, J. D., \& Schriefers, H. (2010). On the flexibility of grammatical advance planning during sentence production: Effects of cognitive load on multiple lexical access. Journal of Experimental Psychology: Learning, Memory, and Cognition, 36(2), 423-440. https://doi.org/10.1037/a0018619

Weeks, J. C., \& Hasher, L. (2014). The disruptive - and beneficial - effects of distraction on older adults' cognitive performance. Frontiers in Psychology, 5. https://doi.org/10.3389/fpsyg.2014.00133

Wheeldon, L. (2013). Producing spoken sentences: The scope of incremental planning. In S. Fuchs, M. Weirich, D. Pape, \& P. Perrier (Eds.), Speech production and perception: Speech planning and dynamics (pp. 97-118). Peter Lang Academic Publishers.

Wheeldon, L., Meyer, A. S., \& Smith, M. (2003). Incremental language production. In L. Nadel (Ed.), Encyclopedia of cognitive science (pp. 760-764). Nature Publishing Group. http://onlinelibrary.wiley.com/doi/10.1002/0470018860.s00252/abstract

Wheeldon, L., Ohlson, N., Ashby, A., \& Gator, S. (2013). Lexical availability and grammatical encoding scope during spoken sentence production. The Quarterly Journal of Experimental Psychology, 66(8), 1653-1673. https://doi.org/10.1080/17470218.2012.754913

Wingfield, A., \& Grossman, M. (2006). Language and the aging brain: Patterns of neural compensation revealed by functional brain imaging. Journal of Neurophysiology, 96(6), 2830-2839. https://doi.org/10.1152/jn.00628.2006

Yang, J.-C., \& Yang, Y.-F. (2008). Horizontal flow of semantic and phonological information in Chinese spoken sentence production. Language and Speech, 51(3), 267-284. https://doi.org/10.1177/0023830908098543 